\title{
Green synthesis of silver nanoparticles mediated by traditionally used medicinal plants in Sudan
}

\author{
Reem Hassan Ahmed ${ }^{1}$ - Damra Elhaj Mustafa ${ }^{2}$ D
}

Received: 26 March 2019 / Accepted: 21 October 2019 / Published online: 10 December 2019

(c) The Author(s) 2019

\begin{abstract}
Sudan has a tremendous wealth flora due to its unique geographical location and diverse climate. Vast records of plants and plants' secondary metabolites are reported to possess redox capacity and can be exploited for the biosynthesis of nanoparticles. Plant-mediated synthesis of silver nanoparticles is preferred due to their availability and their various metabolites. The present review explores the potentiality and diversity of biological activities of silver nanoparticles that originated from the combination of silver and phyto-constituents of mostly traditionally used Sudanese medicinal and aromatic plants. The green synthesis methods of silver nanoparticles mediated by more than 45 traditionally used medicinal plants are critically reviewed. In addition, parameters that affect the synthesis of plant-mediated silver nanoparticles, their characterization techniques and various biological activities are summarized and discussed. Thus, the study of green synthesis of silver nanoparticles and its applications can be extended to involve vast plant diversity of Sudan.
\end{abstract}

Keywords Green synthesis · Characterization $\cdot$ Silver nanoparticles $\cdot$ Medicinal plants

\section{Introduction}

Nanoparticles that are defined to have at least their dimensions in the range of 1-100 nm have received steadily growing interest as a result of their unusual properties, arrangement to form superstructures and applications superior to their bulk counterparts. The nanoparticles are unlike bulk counterparts; their characteristics properties are governed by the rules of quantum mechanics rather than classical physics [1]. Silver nanoparticles, in particular, have been known to fascinate people since Middle Ages because of their unique properties and applications [2].

At present, silver nanoparticles have received high attention due to their extraordinary biological activities. They are used in drug delivery, bio-labeling, sensing, food preservation, wound healings, water purifications and cosmetics.

Damra Elhaj Mustafa

damrj@bahri.edu.sd; damrjm@yahoo.com

1 Department of Biology and Biotechnology, College of Applied and Industrial Sciences, University of Bahri, P. O. Box 12327, Khartoum North, Sudan

2 Department of Chemistry, College of Applied and Industrial Sciences, University of Bahri, P. O. Box 12327, Khartoum North, Sudan
Moreover, silver nanoparticles have other interesting applications such as textiles, electronics, catalysis and paints [3].

Recently, plant-mediated green synthesis of silver nanoparticles is developing into a new and important branch of nanotechnology. It has emerged and gained importance because it is eco environmental and effective cost, with lesser toxicity when related to chemical hazards [4]. Among physical and chemical nanoparticles synthesis, green synthesis has several advantages. For example (1) less toxic and hazardous materials and environmentally benign solvents, (2) simple, rapid and cost effective, (3) consumes less energy and performs under moderate operation conditions, (4) combines the potency of both silver nanoparticles and plant active ingredients. In this respect, plant-mediated silver nanoparticles have been reported to possess more biological activities than nanoparticles synthesized by chemical methods [5].

Previous studies stated the use of different plant parts such as leaf, root, stem, bark, fruit, bud and latex for the synthesis of silver nanoparticles. Plant mediated synthesis of silver nanoparticles is preferred over microbe-mediated synthesis. The latter is not feasible and requires high aseptic conditions, time taking process and long incubation periods. In addition, the reduction properties of plants secondary metabolites are attributed to the higher potential 
ability of plant extracts to synthesize nanoparticles with improved characteristics [6]. In the synthesis of silver nanoparticles, plant extracts and microbes act as reducing agent for reducing $\mathrm{Ag}^{+}$to $\mathrm{Ag}^{0}$ and capping or stabilizing agents for preventing the aggregation of the nanoparticles.

The diversity of climates in Sudan results in a wide range of ecological habitats and vegetation zones. This diverse climatic condition holds the potential of an immense wealth of flora. The present review article reveals the current knowledge about the potential biosynthesis of silver nanoparticles from plants extracts and presents a database that could benefit researchers on their future work regarding the green synthesis of silver nanoparticles. Authors summarize and compare the synthesis procedures by Sudanese medicinal plants, parameters, characterizations and biological activities of silver nanoparticles.

\section{Methods of silver nanoparticles green synthesis}

The green synthesis of silver nanoparticles proceeds through the reduction of silver ions by the phytochemicals as initial step in the nanoparticles formation. The phytochemicals also involved in the subsequent steps by stabilization and directing the shape and size of nanoparticles [7].

The green synthesis of silver nanoparticles depends on several parameters such as concentration of substrate, temperature, reaction time and $\mathrm{pH}$. Importantly, these reaction parameters affect the silver nanofabrication such as size, shape and distribution. The most important factors are summarized and discussed as follows.

\section{Silver ion concentration}

In the present review, the most frequently reported silver ion concentration was $1 \mathrm{mM}$. However, other concentrations were reported. Typically, the following concentrations of silver ions were used 1.75, 2, 3, 5, 8, 10, 53, 100 and $200 \mathrm{mM}$ [8-17]. Employing high concentration of silver ions is probably to shorten the reaction time for the nanoparticle's formation. This is especially important if the amount of reducing phytochemicals in plant extract is not high enough. Interestingly, other researchers have examined and optimized the effect of changing concentrations of silver ions on the morphologies and applications of nanoparticles. Specifically, concentration sets of (0.1, $0.5,1$, and $2 \mathrm{mM}),(0.5,1,2 \mathrm{mM}),(20,50,100 \mathrm{mM})$ and (1-5 mM) were used by several authors [18-21].

\section{Concentration of plants metabolites reducing agents}

It is assumed that the phyto-constituents which are responsible for the reduction of silver ions are tannins, terpenoids, flavonoids, ketones, aldehydes, amides, and carboxylic acids [22]. These constituents are capable of donating electron for the reduction of $\mathrm{Ag}^{+}$ions to $\mathrm{Ag}^{0}$ [23]. Tannins were found to play a key role in the reduction and capping of silver nanoparticles [24]. Other Water-soluble secondary metabolites, most likely proteins, were mainly responsible for the biosynthesis of silver nanoparticles as in Foeniculum vulgare [25].

The effects of changing the concentrations of plant extract have been studied extensively. Many researchers have found that the concentrations of plant extract affect largely the shape and size and hence the nanoparticles biological activities [13, 14, 18, 19, 21, 26, 27].

\section{The effect of temperature}

Synthesis of silver nanoparticles is largely affected by temperature. Mostly, it had been carried out at room temperature as a simple and natural method. In the reviewed articles, synthesis procedures follow the same pattern. It is evident that the stability of plant metabolites requires working at ambient temperature. However, to shorten the synthesis time and enhance complete conversion of $\mathrm{Ag}^{+}$to $\mathrm{Ag}^{0}$, some researchers have attempted synthesis at higher temperatures [8, 9, 14, 28-33]. Interestingly, Krishnan et al. has synthesized silver nanoparticles from Piper nigrum at room temperature, 40,60 and $80^{\circ} \mathrm{C}$ and studied the surface plasmon resonance (SPR) of the resultant nanoparticles by UV spectroscopy and found that nanoparticles synthesized at $80{ }^{\circ} \mathrm{C}$ gave an intense surface plasmon resonance band [28]. An intense SPR band would indicate the formation of high amount of nanoparticles. Microwave irradiation has also been employed to synthesize silver nanoparticles from Tamarind indica [12] and Eucalyptus globulus [34].

\section{Effect of pH}

$\mathrm{pH}$ has an important effect on the fabrication of nanoparticles in many ways such as altering the charge on the metabolites and hence affect the redox reaction and binding between metal and the phytochemical capping agents. Consequently, the shape and size of the nanoparticles are affected by acidity and basicity of the reaction medium. Moreover, the stability of nanoparticles is also sensitive to medium $\mathrm{pH}$ [7]. In spite of the key role, few researchers have considered $\mathrm{pH}$ in their synthesis of nanoparticles. Muthu and Priya studied the effect of changing $\mathrm{pH}$ on the synthesis of silver nanoparticles 
[35]. They found that large-sized nanoparticles were formed at low $\mathrm{pH}$; whereas high dispersed and small-sized nanoparticles at high $\mathrm{pH}$. Similarly, Khalil et al. revealed that the rate of formation of silver nanoparticles increased at high $\mathrm{pH}$ [36]. Stability of the synthesized silver nanoparticles colloid at pH 4 was also reported [19]. Similarly, Krishnan et al. described the stability of silver nanoparticles under different $\mathrm{pH}(2-9)$ and found no effect on the nanoparticles morphology and their SPR peak [28]. Interestingly, Sahni et al. have reported formation of monodisperse silver nanoparticles by addition of ammonia [37].

\section{Effect of reaction time}

Less time taking and mild processes are the most obvious advantages of plant-mediated synthesis of silver nanoparticles. Usually, brown color change takes place immediately after mixing the $\mathrm{Ag}^{+}$ions with plant extracts indicating formation of the nanoparticles. However, reaction time is an important factor for smooth formation of nanoparticles and their shapes, sizes and stabilities. For example, the size of the nanoparticles increases with time [11]. The reaction time is varied according to the synthesis conditions; such as concentration and type of plant extracts, reaction temperatures and $\mathrm{pH}$. Generally, the reaction requires short periods as indicated by most of reviewed works. However, some researchers reported several days for a complete conversion of $\mathrm{Ag}^{+}$and stabilization of the nanoparticles $[11,18]$. Some researchers have monitored the SPR band by UV-vis spectrophotometer and reported noticeable enhancement over time [21, 24]. In contrast, Sathyavathi et al. found blue peak shift in the absorbance from 440 to $427 \mathrm{~nm}$ with increasing reaction time [38]. The blue shifted band indicates particle size reduction, which is probably due to gradual oxidation of nanoparticles.

\section{Separation and characterization of silver nanoparticles}

The purification of silver nanoparticles by centrifugation technique is widely used to get rid of unreacted materials and by products. Proper separation and purification are highly required for the nanoparticles characterizations and applications [7, 23]. Various characterization techniques can be used to identify the shape, size, surface and dispersity of the nanoparticles. The common useful methods include UV-visible spectrophotometry, dynamic light scattering, scanning electron microscopy, transmission electron microscopy, zeta potential, Fourier transform infrared spectroscopy, X-ray diffraction, energy dispersive spectroscopy, auger electron spectroscopy, scanning tunneling microscopy, atomic force microscopy. In the current review, results obtained from the most important techniques will be summarized and discussed.

\section{UV-visible spectrophotometry}

UV-visible spectrophotometry is a readily available technique allows fast identification and characterization of silver nanoparticles. It gives strong absorbance band known as surface plasmon resonance (SPR) in the range $400-500 \mathrm{~nm}$ due to the interaction between light and mobile surface electrons of silver nanoparticles [16, 39, 40].

In the current work, most of SPR peaks are within the anticipated wavelength range, however, some researchers have reported SPR peaks below $400 \mathrm{~nm}$ for their synthesized nanoparticles [9, 15, 31, 41, 42]. The absorbance bands below $400 \mathrm{~nm}$ are probably due to absorption of silver ions, complexes, impurities and plant phytochemicals. The characteristic of SPR is largely affected by the shape and size of the nanoparticles and dielectric constant of the surrounding medium [43]. The high sensitivity of SPR to the change of surrounding medium could be the reason for having wide range variations of absorption bands.

In the present work, the examination of the particle sizes and SPR peaks did not result in a clear correlation when data in Table 1 were analyzed. This is probably due to variation in the synthesis conditions and type of plant extracts. However, proportional relationships between size and SPR peaks have been observed by examining the silver colloids synthesized using different concentrations of plant extract or silver ions. Specifically, Roy et al. reported SPR peaks of 420 and $430 \mathrm{~nm}$ for particles with diameters of 65.67 and 66.98 , respectively [26]. Similarly, changing of silver ions concentrations in the range 0.02 to $0.1 \mathrm{M}$ have been found to result in nanoparticles with SPR peaks and diameters that increase with decreasing the silver ions concentrations [20]. Other researchers, synthesized silver nanoparticles using varied plant extract concentrations and reported a blue shift of SPR peak and decrement of particle size with increasing the amount of plant extract $[13,36,44]$.

Obviously, the green synthesis resulted in various shapes and sizes dominated by spherical, polydisperse and have been found to be efficient for silver nanoparticles formation as seen in the reviewed articles. However, it is clear that the green synthesis results in less controllable morphologies compared to the physical and chemical synthesis methods. This is probably due to the presence of different reducing/ capping phytochemicals that make multi rates of redox reaction and growing processes of the silver nanoparticles.

\section{Fourier transforms infrared spectroscopy (FTIR)}

The surface chemistry of the nanoparticles is revealed by FTIR. This technique helps to identify the functional groups 
Table 1 Green synthesis of silver nanoparticles using plants extracts

\begin{tabular}{|c|c|c|c|c|c|}
\hline Plant name & Part of plant & Characterization techniques & NP size (nm) & SPR peak (nm) & Reference \\
\hline Abutilon indicum & Leaf & UV, DLS, EDX, TEM, SEM, XRD & $5-25$ & 455 & {$[24]$} \\
\hline Acacia nilotica & Leaf & UV, EDX, FTIR, SEM & $30-150$ & 450 & {$[45]$} \\
\hline \multirow[t]{2}{*}{ Acacia senegal } & Leaf & UV, TEM, XRD, FTIR & $10-19$ & 467 & [46] \\
\hline & Gum & UV, AFM, XRD, FTIR & $81.45 \pm 2.07$ & 421 & [14] \\
\hline Acacia seyal & Gum & UV, AFM, XRD, FTIR & $81.45 \pm 2.07$ & 421 & [14] \\
\hline Acalypha hispida & Leaf & UV, XRD. FTIR, TEMGCMS & $20-50$ & 424 & [8] \\
\hline Adansonia digitata & Fruit, leaf & UV, FTIR, XRD, AFM, SEM, EDX, TEM & $5-64$ & 431 & {$[33]$} \\
\hline \multirow[t]{2}{*}{ Allium сера } & Fruit & TEM, ZPM, FTIR & $14.8 \pm 3.2$ & 436 & [47] \\
\hline & Bulb & UV, TEM & 10 & 401 & {$[37]$} \\
\hline \multirow[t]{2}{*}{ Allium sativum } & Fruit & UV, SEM, TEM, XRD, FTIR & $3-6$ & 375 & [15] \\
\hline & Fruit & TEM, ZPM, FTIR & $47.2 \pm 14.8,7.4 \pm 3$ & 428 & [47] \\
\hline \multirow[t]{2}{*}{ Azadirachta indica } & Leaf & UV, DLS, TEM, & 34 & $436-446$ & {$[21]$} \\
\hline & Leaf & UV, DLS & $65.67,66.98$ & $420-450$ & [26] \\
\hline Calatropis procera & Latex & UV, TEM, XRD, FTIR, LDPSA & 12.33 & 290 & [9] \\
\hline Capparis decidua & Stem & UV, TEM, FTIR, & $1-19$ & 460 & [48] \\
\hline Capsicum frutescens & Fruit & UV, SEM, TEM, XRD, FTIR & $3-18$ & 480 & [15] \\
\hline Carum carvi & Leaf & TEM, SEM, EDS, XRD & 10 & 450 & [17] \\
\hline Fagonia cretica & Whole plant & UV, HPLC, FTIR, TEM & 16 & 440 & [19] \\
\hline Jatropha curcas & Leaf & $\begin{array}{l}\text { UV, SEM, EDX, TEM, AFM, FTIR, XRD, } \\
\text { AE }\end{array}$ & $20-100$ & 430 & {$[10]$} \\
\hline \multirow[t]{2}{*}{ Cassia auriculata } & Leaf & UV, XRD, TEM & $10-35$ & 435 & {$[35]$} \\
\hline & Leaf & UV, FTIR, SEM, EDAX, XRD, TEM & $50-100$ & 452 & {$[27]$} \\
\hline Cassia occidentalis & Leaf & UV, TEM, SEM, XRD, EDX & 31 & 461 & [49] \\
\hline Catharanthus roseus & Leaf & UV, XRD, FTIR, EDX, SEM & $35-55$ & 423 & {$[50]$} \\
\hline Citrus sinensis & Peel & UV, XRD, AFM, FTIR & 34 & 420 & {$[51]$} \\
\hline Coffee arabica & Seed & UV, XRD, TEM, SEM-EDX, FTIR, DLS & $10-40,10-50,20-150$ & $447,459,445$ & {$[20]$} \\
\hline \multirow[t]{2}{*}{ Coriandrum sativum } & Seed & UV, XRD, FTIR, SEM, & 13.09 & 421 & [16] \\
\hline & Leaf & UV, XRD, FTIR, TEM & 26 & 427 & {$[38]$} \\
\hline Cyperus rotundus & Whole plant & UV, FTIR, SEM, EDX & $20.5 \pm 9.6$ & 446 & {$[52]$} \\
\hline Datura stramonium & Leaf & UV, FTIR, TEM, XRD & 18 & 444 & {$[53]$} \\
\hline Eucalyptus globulus & Leaf & UV, FTIR, XRD, TEM, SEM, EDX, TGA & $1.9-4.3,5-25$ & 428 & {$[34]$} \\
\hline Fagonia cretica & Fruit & UV, HPLC, FTIR, TEM & 16 & 440 & [19] \\
\hline Foeniculum vulgare & Seed & UV, SEM, FTIR & $11-25$ & 475 & {$[25]$} \\
\hline Hibiscus sabdariffa & Flower & TEM, SEM, EDX, XRD,FTIR & 3.9 & - & {$[54]$} \\
\hline Jatropha curcas & Leaf & UV, SEM, EDX, TEM, AFM, FTIR, XRD & $20-200$ & 430 & {$[10]$} \\
\hline Kigelia Africana & Fruit & UV, FTIR, XRD, SEM, EDX, TEM & 10 & $285-350$ & {$[31]$} \\
\hline \multirow[t]{2}{*}{ Lantana camara } & Leaf & XRD, SAED, XPS, FTIR, AFM, TEM & $24-11,34-20,31-17,27-14$ & $436,421,413,400$ & {$[44]$} \\
\hline & Leaf & UV, FTIR, XRD & - & 439 & {$[55]$} \\
\hline Lawsonia inermis & Leaf & UV, FTIR, TEM, XRD & 30 & 445 & {$[56]$} \\
\hline \multirow[t]{2}{*}{ Magnifera indica } & Leaf & XRD, LDPSA, SEM, EDS, UV & 31.7 & 393 & {$[42]$} \\
\hline & Leaf & UV, FTIR, SEM & 100 & 420 & {$[57]$} \\
\hline Mentha piperta & Leaf & UV, SEM, TEM & 35 & 420 & {$[58]$} \\
\hline \multirow[t]{2}{*}{ Moringa oleifera } & Leaf & TEM, SEM, FTIR & 9,11 & 450,440 & [59] \\
\hline & & UV, TEM & 57 & $430-440$ & {$[30]$} \\
\hline Nigella sativa & Seed & UV, ZPM, FTIR, TEM & $10-20$ & 432 & [40] \\
\hline Olea europaea $L$. & Leaf & UV, FTIR, SEM, TGA & $20-25$ & 441 & {$[36]$} \\
\hline Phoenix dactylifera & Leaf & SEM, XRD & $30-85$ & $439.5,447$ & [13] \\
\hline \multirow[t]{2}{*}{ Phyllanthus amarus } & Leaf & UV, FTIR, XRD, TEM & 36 & 421 & {$[60]$} \\
\hline & Whole plant & UV, FTIR, TEM, DLS & 33.7 & 420 & [29] \\
\hline Pimpinella anisum & Seed & TEM, EDS, XRD, UV & 8.3 & 442 & {$[11]$} \\
\hline
\end{tabular}


Table 1 (continued)

\begin{tabular}{llllll}
\hline Plant name & Part of plant & Characterization techniques & NP size (nm) & SPR peak (nm) & Reference \\
\hline Piper nigrum & Fruit & UV, FTIR, TEM & 20 & 441 & {$[28]$} \\
Ricinus communis & Leaf & UV, UHRTEM, SAED, XRD, FTIR, TGA & $30-40$ & 10 & 430,442 \\
Tamarindus indica & Fruit & UV, XRD, SEM, EDX, TEM & 35.4 & 432 & $420-430$ \\
Tinospora cordifolia & Whole plant & UV, FTIR, DLS & $16-28$ & 435 & {$[61]$} \\
Tribulus terrestris & Fruit & TEM, AFM, XRD, FTIR, UV & $70-110$ & 430 & {$[12]$} \\
Withania somnifera & Leaf & UV, LDA, TEM, SEM, XRD, AFM & $12-36$ & 430 & {$[62]$} \\
& & UV, SEM, TEM, XRD & $3-22$ & $400-480$ & {$[63]$} \\
Zingiber officinale & Rhizome & UV, SEM, TEM, XRD, FTIR & {$[15]$} \\
\hline
\end{tabular}

$U V$ UV-visible spectrophotometry, FTIR Fourier transforms infrared spectroscopy, SEM scanning electron microscopy, TEM transmission electron microscopy, $X R D X$ X-ray diffraction, $D L S$ dynamic light scattering, $A F M$ atomic force microscopy, $L D P S A$ laser diffraction particle size analyzer, $E D S$ energy dispersive spectrometry, $Z P M$ zeta potential measurement, $E D X X$-ray energy dispersive spectrophotometer, $X P S \mathrm{X}$-ray photoelectron spectroscopy, $T G A$ thermogravimetric analysis, $S A E D$ selected area electron diffraction, $L D A$ laser doppler anemometry, GCMS Gas chromatography-mass spectrometry, HPLC high performance liquid chromatography, $A E$ atomic emission

of both plant extracts and the resulting silver nanoparticles. Table 2 summarizes the FTIR data of the reviewed articles. It is important to note that the FTIR technique is used at least to characterize the synthesized silver nanoparticles. Examining FTIR of the plant phytochemicals in free form or bounded to silver nanoparticles sometimes reveals slight band shifts as shown in Table 2. These shifts are taken as an evidence for the metal reduction and formation of nanoparticles.

The most important phytochemical constituents responsible for the reduction and capping of silver nanoparticles as revealed by FTIR and phytochemical studies are alkaloids, flavonoids, tannins, terpenes and quinones [64]. Table 3 summarizes the phytochemical constituents of the reviewed plants. Obviously, within a plant extract there are different reducing and capping agents. Thus, most of silver nanoparticles mediated by plant extract result in polydisperse and several shapes and sizes. Few research articles studied the employment of pure phytochemical compounds on silver nanoparticles synthesis and applications. In this respect, Jain and Mehata reported synthesis of silver nanoparticles using quercetin [65]. The use of pure phytochemical compounds on the synthesis of nanoparticles may probably control the nanoparticles morphology and results into new scopes for nanoparticles applications.

\section{Microbe-mediated silver nanoparticles synthesis}

As mentioned earlier, the microbe-mediated synthesis is not preferred and less common. In this respect there are few reports on the synthesis mediated by yeast, fungi, bacteria, algae and viruses [66]. Microbe-mediated synthesis involves the bio-reduction of metal salts to elemental metal. Fungi such as Aspergillus fumigates, Fusarium oxysporum,
Trichoderma reesei and Coriolus versicolor have been used for the synthesis of silver nanoparticles [67]. Another study reported the synthesis of silver nanoparticles from Mushroom polysaccharides [68]. Interestingly silver nanoparticles produced by pellet of Escherichia coli showed a marked activity against Salmonella typhi, Bacillus subtilis, Klebsiella pneumonia and Vibrio cholera [69]. Alternatively, biological molecules such as nucleic acids have also been reported to mediate silver nanoparticles synthesis by acting as reducing agents [66].

\section{Biological activity}

The present review article reveals the current knowledge about the biosynthesis of silver nanoparticles from plants extracts and their potential biological activities.

\section{Anti-microbial activity}

Anti-microbial effect of silver nanoparticles has been widely investigated, yet their mode of actions is not fully elucidated. This activity was attributed to many factors summarized as small size of the nanoparticles and increased surface area provide opportunities for interactions with bacterial cell because it leads to increased membrane permeability and cell destruction to bacteria and fungi. Silver nanoparticles can cause cell breaking down and changes in the cell membrane permeability. In addition, silver nanoparticles attach the surface of the cell membrane, penetrating in bacteria and disturb the cell function, interactions of silver nanoparticles with amino acids and enzymes: bonding with amino acids (especially to $-\mathrm{SH}$ group), generation of ROS. It is also attributed to the fact that cells are majorly made up of sulfur and phosphorus which are soft bases and DNA has sulfur and phosphorus as its major components; silver 
Table 2 Comparison of FTIR data of selected plant extracts and plant synthesized silver nanoparticles

\begin{tabular}{|c|c|c|c|c|}
\hline \multirow[t]{2}{*}{ Plant name } & \multicolumn{2}{|c|}{ FTIR absorption bands $\left(\mathrm{cm}^{-1}\right)$} & \multirow[t]{2}{*}{ Possible functional group } & \multirow[t]{2}{*}{ References } \\
\hline & Plant extract & $\mathrm{AgNP}$ & & \\
\hline \multirow[t]{5}{*}{ Abutilon indicum } & 3321 & 3290 & $-\mathrm{OH}$ & \multirow[t]{5}{*}[24]{} \\
\hline & 2925 & 2931 & $\mathrm{C}-\mathrm{H}$ & \\
\hline & 1627 & 1653 & $\mathrm{~N}-\mathrm{H}$ (amide I) & \\
\hline & 1393 & 1383 & $\mathrm{C}=\mathrm{O}$ & \\
\hline & 1114 & 1064 & $\mathrm{C}-\mathrm{O}$ & \\
\hline \multirow[t]{3}{*}{ Capparis decidua } & 3274 & 3279 & $\mathrm{~N}-\mathrm{H}$ & \multirow[t]{3}{*}[48]{} \\
\hline & 1636 & 1637 & $\mathrm{C}=\mathrm{C}$ (alkene, aromatic) & \\
\hline & 524 & 549 & $\mathrm{C}-\mathrm{Br}$ & \\
\hline \multirow[t]{6}{*}{ Cassia auriculata } & 3393 & 3406 & $\mathrm{O}-\mathrm{H}$ & \multirow[t]{6}{*}[35]{} \\
\hline & 2925 & 2925 & $\mathrm{C}-\mathrm{H}$ & \\
\hline & 1629 & - & $\mathrm{C}=\mathrm{O}$ & \\
\hline & 1605 & 1602 & $\mathrm{C}=\mathrm{C}$ & \\
\hline & 1389 & 1389 & Amide, $\mathrm{NO}_{3}$ of $\mathrm{AgNO}_{3}$ & \\
\hline & 1068 & 1082 & $\mathrm{C}-\mathrm{O}$ & \\
\hline \multirow[t]{11}{*}{ Coffea arabica } & $876-880$ & $780-1100$ & $\mathrm{C}-\mathrm{C}, \mathrm{C}-\mathrm{OH}, \mathrm{C}-\mathrm{H}$ (ring), $\mathrm{C}-\mathrm{CO}$ & \multirow[t]{11}{*}[20]{} \\
\hline & $1036-1150$ & - & $\mathrm{C}-\mathrm{O}, \mathrm{C}-\mathrm{O}-\mathrm{C}, \mathrm{C}-\mathrm{C}$ & \\
\hline & 1408 & - & $\mathrm{COO}-$ & \\
\hline & 1517 & - & $\mathrm{C}=\mathrm{C}$ & \\
\hline & 1603 & - & $\mathrm{COO}-$ & \\
\hline & $1650-1550$ & $1597-1630$ & $\mathrm{C}=\mathrm{O}$ & \\
\hline & 1639 & 1632 & $\mathrm{~N}-\mathrm{H}, \mathrm{C}=\mathrm{O}, \mathrm{C}-\mathrm{C}, \mathrm{C}-\mathrm{N}$ & \\
\hline & 1744 & 1742 & COO- (ester) & \\
\hline & $2800-3500$ & $3420-3770$ & $\mathrm{O}-\mathrm{H}$ & \\
\hline & 2925 & 2960 & $\mathrm{C}-\mathrm{H}(\mathrm{CH} 3)$ & \\
\hline & 2855 & 2850 & $\mathrm{C}-\mathrm{H}(-\mathrm{CH} 2)$ & \\
\hline \multirow[t]{6}{*}{ Cyperus rotundus } & 3363 & - & $\mathrm{O}-\mathrm{H}$ & \multirow[t]{6}{*}[52]{} \\
\hline & 2976 & 2976 & $\mathrm{C}-\mathrm{H}$ & \\
\hline & 1715 & 1715 & $\mathrm{C}=\mathrm{O}$ & \\
\hline & 1248 & 1248 & $\mathrm{C}-\mathrm{O}$ & \\
\hline & 1099 & 1099 & $\mathrm{P}-\mathrm{O}$ & \\
\hline & 726 & 726 & $\mathrm{~N}-\mathrm{H}$ & \\
\hline \multirow[t]{7}{*}{ Eucalyptus globulus } & 3437 & 3437 & $\mathrm{O}-\mathrm{H}$ & \multirow[t]{7}{*}[34]{} \\
\hline & 2927 & 2927 & $\mathrm{C}-\mathrm{H}$ & \\
\hline & 1743 & 1743 & $\mathrm{C}=\mathrm{O}$ & \\
\hline & 1607 & 1607 & $\mathrm{C}=\mathrm{C}$ & \\
\hline & 1382 & 1382 & $\mathrm{O}-\mathrm{H}$ & \\
\hline & 1247 & 1247 & $\mathrm{C}-\mathrm{O}-\mathrm{H}$ & \\
\hline & 1060 & 1060 & $\mathrm{C}-\mathrm{O}$ & \\
\hline \multirow[t]{5}{*}{ Euphorbia hirta } & 3312 & 3340 & $\mathrm{O}-\mathrm{H}$ & \multirow[t]{5}{*}{ [52] } \\
\hline & 2921 & 2921 & $\mathrm{C}-\mathrm{H}$ & \\
\hline & 1715 & 1715 & $\mathrm{C}=\mathrm{O}$ & \\
\hline & 1100 & 1100 & $\mathrm{P}-\mathrm{O}$ & \\
\hline & 726 & - & $\mathrm{N}-\mathrm{H}$ & \\
\hline
\end{tabular}


Table 2 (continued)

\begin{tabular}{|c|c|c|c|c|}
\hline \multirow[t]{2}{*}{ Plant name } & \multicolumn{2}{|c|}{ FTIR absorption bands $\left(\mathrm{cm}^{-1}\right)$} & \multirow[t]{2}{*}{ Possible functional group } & \multirow[t]{2}{*}{ References } \\
\hline & Plant extract & $\mathrm{AgNP}$ & & \\
\hline \multirow[t]{13}{*}{ Fagonia cretica } & 3864.44 & - & $\mathrm{O}-\mathrm{H}$ & \multirow[t]{13}{*}[19]{} \\
\hline & 3729.37 & 3739.15 & $\mathrm{O}-\mathrm{H}$ & \\
\hline & 3626.26 & - & $\mathrm{O}-\mathrm{H}$ & \\
\hline & 3467.80 & - & $\mathrm{N}-\mathrm{H}$ & \\
\hline & 2916.00 & - & $\mathrm{C}-\mathrm{H}$ & \\
\hline & 2358.74 & - & $\mathrm{N}-\mathrm{H}$ & \\
\hline & 1636.56 & - & $\mathrm{C}=\mathrm{O}$ & \\
\hline & 1472.89 & - & $\mathrm{N}-\mathrm{H}$ & \\
\hline & 1401.80 & - & $\mathrm{C}-\mathrm{O}-\mathrm{H}$ & \\
\hline & 1114.29 & - & $\mathrm{C}-\mathrm{O}-\mathrm{C}$ & \\
\hline & 1061.73 & 1050.90 & $\mathrm{C}-\mathrm{O}$ & \\
\hline & 869.08 & - & $\mathrm{C}-\mathrm{C}, \mathrm{C}-\mathrm{OH}, \mathrm{C}-\mathrm{H}$ (ring) & \\
\hline & 627.47 & 643.89 & $\mathrm{C}-\mathrm{H}$ bending & \\
\hline \multirow[t]{5}{*}{ Olea europaea } & 3409 & 3395 & $\mathrm{O}-\mathrm{H}$ & \multirow[t]{5}{*}[36]{} \\
\hline & 1733 & - & $\mathrm{C}=\mathrm{O}$ & \\
\hline & 1624 & - & Amide I carbonyl & \\
\hline & 1077 & - & $\mathrm{C}-\mathrm{N}$ & \\
\hline & 651 & - & $=\mathrm{C}-\mathrm{H}$ bend & \\
\hline \multirow[t]{9}{*}{ Phoenix dactylifera } & 3330 & 3330 & $\mathrm{~N}-\mathrm{H}, \mathrm{O}-\mathrm{H}$ & \multirow[t]{9}{*}[13]{} \\
\hline & 2976 & 2976 & $\mathrm{C}-\mathrm{H}$ & \\
\hline & 2128 & 2128 & $\mathrm{C}-\mathrm{O}$ & \\
\hline & 1658 & 1635 & $\mathrm{C}=\mathrm{O}$ & \\
\hline & 1453 & 1453 & $\mathrm{C}=\mathrm{C}$ & \\
\hline & 1377 & 1377 & $\mathrm{C}-\mathrm{N}$ & \\
\hline & 1278 & 1278 & $\mathrm{C}-\mathrm{O}$ & \\
\hline & 1052 & 1052 & $\mathrm{C}-\mathrm{O}$ & \\
\hline & 873 & 873 & $=\mathrm{C}-\mathrm{H}$ & \\
\hline \multirow[t]{10}{*}{ Piper nigrum } & 3416 & 3421 & $\mathrm{O}-\mathrm{H}$ & \multirow[t]{10}{*}[28]{} \\
\hline & 2921 & - & $\mathrm{C}-\mathrm{H}$ & \\
\hline & 2388 & - & $\mathrm{C}-\mathrm{H}$ & \\
\hline & 1625 & 1645 & $\mathrm{C}=\mathrm{O}$ & \\
\hline & 1388 & - & $\mathrm{N}=\mathrm{O}$ & \\
\hline & - & 1230 & $\mathrm{C}-\mathrm{N}$ & \\
\hline & 1221 & - & $\mathrm{C}-\mathrm{F}$ & \\
\hline & 1023 & - & $\mathrm{C}-\mathrm{O}$ & \\
\hline & 565 & - & $\mathrm{C}-\mathrm{I}, \mathrm{OH}$ bending & \\
\hline & 526 & - & $\mathrm{C}-\mathrm{I}, \mathrm{OH}$ bending & \\
\hline \multirow[t]{6}{*}{ Tamarind indica } & 3384 & 3398 & $\mathrm{O}-\mathrm{H}$ & \multirow[t]{6}{*}[12]{} \\
\hline & 2941 & 2940 & $\mathrm{C}-\mathrm{H}$ & \\
\hline & 1730 & 1740 & $\mathrm{C}=\mathrm{O}$ & \\
\hline & - & 1632 & $\mathrm{C}=\mathrm{C}$ aliphatic & \\
\hline & 1406 & 1415 & $\mathrm{C}=\mathrm{C}$ aromatic & \\
\hline & 1069 & 1075 & $\mathrm{C}-\mathrm{O}$ (ester) & \\
\hline
\end{tabular}


Table 2 (continued)

\begin{tabular}{lllll}
\hline Plant name & \multicolumn{2}{l}{ FTIR absorption bands $\left(\mathrm{cm}^{-1}\right)$} & Possible functional group & References \\
\cline { 2 - 3 } & Plant extract & AgNP & & \\
\hline Tribulus terrestris & 3489 & 3419 & O-H & [62] \\
& 2811 & 2812 & C-H & \\
2728 & 2726 & C-H & \\
2164 & 2171 & C $=$ C & C $=$ C \\
1591 & 1613 & C-N & C-N aromatic amine \\
1388 & - & 1349 & C-N aliphatic amine \\
1349 & 1125 &
\end{tabular}

nanoparticles can act on these soft bases and destroy the DNA which would definitely lead to cell death.

In the current review, more than 35 medicinal plants have proven to show significant anti-microbial activities against various Gram-positive and Gram-negative bacteria as well as anti-fungal effects. It is reported that green silver nanoparticles showed more efficient anti-microbial activity than the plant extract alone as in cases of Azadirachta indica [21], Calatropis procera [9], Fagonia cretica [19], Tinospora cordifolia [29]. While antimicrobial activity of synthesized silver nanoparticles from Phyllanthus amarus was reported to be higher than that of the standard drug used in the study [29]. Silver nanoparticles from Lawsonia inermis gel in combination with antibiotics showed a synergistic anti-microbial effect [56]. Whereas Dias et al. stated that a cream incorporated with silver nanoparticles biosynthesized from Withania somnifera possessed a significantly higher antimicrobial activity [18].

Some green synthesized silver nanoparticles have exhibited a prominent antifungal activity such as Calatropis procera [9], Lawsonia inermis [56], Phyllanthus amarus, Tinospora cordifolia [29] and Withania somnifera [18]. The actual mechanism behind the antifungal activity of silver nanoparticles is not yet fully understood. However, it is assumed that disrupting the structure of the cell membrane by destructing the membrane integrity could be responsible for this biological action [23]. Nanoparticles synthesized from spice medicinal plants e.g. Allium sativum (garlic), Zingiber officinale (ginger), and Capsicum frutescens (cayenne pepper); which are important spices with well-known medicinal uses; were evaluated. These spices were reported to have various biological activities including antimicrobial and antioxidant activities. Silver nanoparticles synthesized from these spices exhibited broad-spectrum antibacterial activities thus, being suggested as valuable potential alternatives [15]. However, it was noted that the antimicrobial activity of silver nanoparticles against Gram-positive bacteria was reported to be less compared to Gram-negative bacteria and this may be attributed to the presence of the peptidoglycan layer which is negatively charged and prevents the free entry of silver ions into the cell wall of the bacteria [26].

\section{Anticancer activity}

Induced cell death in cancer cells was assumed to be mediated by increased oxidative stress leading to apoptosis of these cells. In the present article, it was found that silver nanoparticles synthesized from Abutilon indicum showed a dose dependent anti-cancer activity against human colon cancer at a very low concentration. Their anticancer activity was attributed to the enhancement of intracellular ROS generation and depletion of mitochondrial membrane potential that leads to further DNA fragmentation and cell cycle arrest [24]. Whereas silver nanoparticles synthesized using Pimpinella anisum seeds has shown cytotoxicity on human neonatal skin stromal cells and colon cancer cells [11]. Biosynthesized silver nanoparticles and Piper nigrum extract showed promising anticancer activity against breast cancer cells (MCF-7) and human pharynx cancer cell line (Hep-2) [28]. Other findings revealed that silver nanoparticles from Nigella sativa seeds were found to be effective against hepatocellular carcinoma using HepG2 cell lines [40].

\section{Anti-oxidant activity and radical scavenging activity}

In biological systems, uncontrolled accumulation of $\mathrm{H}_{2} \mathrm{O}_{2}$ leads to the formation of oxygen free radicals which causes massive damage to cell membranes. The antioxidant ability of green silver nanoparticles could be attributed to the functional groups adhered to them that came from the medicinal plant extracts. Many studies have investigated the anti-oxidant activity of silver nanoparticles from various medicinal plants, e.g. Abutilon indicum [24], Allium sativum, Capsicum frutescens, Cassia occidentalis [49] and Zingiber officinale [15]. 
Table 3 Phytochemical constituents of plants mediated green synthesis of silver nanoparticles

\begin{tabular}{|c|c|c|}
\hline Plant & Phytochemicals & References \\
\hline Acacia nilotica & Flavonoids, tannins, saponins, glycosides, alkaloids, sterols, terpenoids, anthraquinones & [70] \\
\hline Acacia senegal & Saponins, sterols, tannins & [71] \\
\hline Acacia seyal & Flavonoids, alkaloids, tannins, terpenoids, cardiac glycosides & [72] \\
\hline Allium сера & Steroids, alkaloids, tannins, flavonoids, cardiac glycosides, anthraquinones & [73] \\
\hline Allium sativum & Saponins, steroids, tannins, cardiac glycosides & [74] \\
\hline Azadirachta indica & Alkaloids, glycosides, phenols, steroids & [75] \\
\hline Cajanus cajan & Alkaloids, flavonoids, tannins, saponins, terpenes & [76] \\
\hline Calatropis procera & Glycosides, sterols, triterpenes, saponins, cardiac glycosides & [77] \\
\hline Capparis decidua & Alkaloids, glycosides & [78] \\
\hline Capsicum frutescens & Phenolic compounds, active compound (capsaicin) & [79] \\
\hline Carum carvi & Alkaloids, glycosides, sterol, terpenes & [80] \\
\hline Coriandrum sativum & Flavonoids, isocoumarins, fatty acids, sterols, coumarins & [81] \\
\hline Cassia auriculata & Alkaloids, flavonoids, steroids, saponins, tannins & [82] \\
\hline Cassia occidentalis & Flavonoids, phenols, tannins, amino acids, saponins, glycosides, terpenoids, steroids & [83] \\
\hline Catharanthus roseus & Alkaloids, phytosterols, phenolic compounds, tannins & [84] \\
\hline Citrus sinensis & Flavonoids, steroids, hydroxyamides, fatty acids, coumarins, carotenoids & [85] \\
\hline Croton zambesicus & Diterpenes & [86] \\
\hline Cyperus rotundus & Essential oils (active compound cyperone) & [87] \\
\hline Datura stramonium & Tannins, alkaloids & [88] \\
\hline Fagonia cretica & Saponins, cardiac glycosides, tannins & [89] \\
\hline Foeniculum vulgare & 76 volatile components in the essential oil & {$[90]$} \\
\hline Jatropha carcus & Phenols, tannins, phytic acid & [91] \\
\hline Kigelia africana & Iridoids, naphthoquinones, flavonoids, terpenes, phenylethanoglycosides & [92] \\
\hline Lantana camara & Triterpenoids, flavonoids, fixed oil, tannins & [93] \\
\hline Lawsonia inermis & Quinones, phenylpropanoids, flavonoids, terpenoids, phenolic compounds, fatty acids & [94] \\
\hline Magnifera indica & Terpenoids, flavonoids, saponins, tannins & [95] \\
\hline Mentha piperta & Alkaloids, flavonoids, phenols, steroids, tannins & [96] \\
\hline Moringa oleifera & $\begin{array}{l}\text { Phenolic acids, flavonoids, alkaloids, glucosinolates, tannins, saponins, oxalates, phytates, steroids, } \\
\text { triterpenoids, flavonoids, anthraquinones, }\end{array}$ & [97] \\
\hline Nigella sativa & Alkaloids, tannins, flavonoids, sterols & [98] \\
\hline Olea europaea L. & Flavonoids, triterpenes, sterol & [99] \\
\hline Phoenix dactylifera & Phenolic acids, flavonoids and tanins & {$[100]$} \\
\hline Phyllanthus amarus & Saponins, alkaloids, tannins, cardiac glycosides & [101] \\
\hline Ricinus communis & Tannins, saponins, terpenoids, polyuronoids, reducing sugars, flavonoids, alkaloids, anthraquinones & [102] \\
\hline Tamarindus indica & Alkaloid, anthraquinone, saponins, glycosides & [103] \\
\hline Tinospora cordifolia & Terpenoids, steroids, glycosides, flavonoids, phlobatannins & [104] \\
\hline Vernonia amygdalina & Terpenoids, flavonoids, saponins, tannins, alkaloids, cardiac glycosides & [75] \\
\hline Withania somnifera & steroids, phenols, alkaloids, flavanoids, glycosides, tannins and saponins & [105] \\
\hline Zingiber officinale & Alkaloid, flavonoids, glycosides, resins, saponins, sterol terpenes, tannins, carbohydrates & [80] \\
\hline
\end{tabular}
dgnr

\section{Other biological activities of green silver nanoparticles}

Acacia Seyal silver nanoparticles significantly reduced cellular infiltration and granulamatous inflammation in ankle joints tissues of induced arthritic rats. So, it is concluded that gum arabic- silver nanoparticles worked as nano-cargo for enhanced anti-arthritic hesperidin (standard drug) in induced arthritic rats [14].

Anti-plasmodial effect against Plasmodium falciparum was detected using silver nanoparticles from Catharanthus roseus extract [50]. However, no proper mechanism for anti-plasmodial action of silver nanoparticles from this plant was demonstrated. Alternatively, Magnifera indica 
silver nanoparticles enhanced dual dentistry applications and hence can be used for dental restoration [42].

\section{Cytotoxicity}

The effect of silver nanoparticles synthesized from aqueousleaf-extract of Mentha piperita was detected on one of the most important neurological enzymes, i.e. acetylcholinesterase (AChE). It showed that these green synthesis nanoparticles from this plant extract might cause neurotoxicity via inhibiting AChE activity. This activity was confirmed by conduction of enzyme kinetic studies which revealed that silver nanoparticles were capable of binding to both the free AChE enzyme and to the enzyme-substrate (AChE-AChI) complex [58]. Results of this study showed that; due to their small size, nanoparticles may easily cross the blood brain barrier and could interact with various neurological targets, which in turn causes neurotoxic effects. This may draw the attention that even green silver nanoparticles are not safe and could cause some neurotoxicity due to their interaction with AChE. While Cassia occidentalis silver nanoparticles showed lower heomolytic activity (1.7\%) to human blood i.e. less toxicity [49].

\section{Effect of size and shape of the nanoparticles on biological activity}

It is noted that almost all silver nanoparticles of the present plant extracts included in this review attained a spherical shape. Studies showed that spherical shaped silver nanoparticles have high surface to volume ratio to interact with the cell walls of pathogens which gives better antimicrobial activity [33]. Nanoparticles with a size range of 11-15 nm is highly suitable for biomedical application because the size of synthesized nanoparticles is within the tolerable range and will not cause toxicity within the cell [19].

In the current review, it is stated that small size nanoparticles have more penetration power to cell membranes, however, too small size brings the issue of enhanced toxicity compared to larger size nanoparticles, thus, an appropriate size is highly desirable for specified biological applications. Small particle size proves its higher potential antimicrobial activity as in Cassia auriculata [27] and Adansonia digitata [33].

\section{Conclusion}

The current review describes the green synthesis of silver nanoparticles mediated by several medicinal plants. The nanoparticles fabrication conditions, characterizations and biological activities presented a database that could benefit researchers on their future work regarding green synthesis of silver nanoparticles.

Wide range of medicinal plants traditionally used in Sudanese folk medicine has been exploited for the green synthesis of silver nanoparticles as simple, cost-effective, ecofriendly and rapid technique. However, this area is still dormant and more researches are required to explore the potentiality of other Sudanese flora. The silver nanoparticles synthesized using reducing and capping plants extracts are reported to have wide variations in shapes and sizes; which showed an impact on their biological activities. However, more studies are required to elucidate the effect of pure secondary metabolites that may control the morphology of the silver nanoparticles and hence their biological activities and other applications. These kinds of studies could provide better understanding of mechanism and efficiency of silver nanoparticles.

A thorough research on Sudan flora is in way to exploit green synthesized silver nanoparticles. This would be associated with a possible warranty on their safety and realization of their full potentiality in the era of green nanotechnology.

Open Access This article is licensed under a Creative Commons Attribution 4.0 International License, which permits use, sharing, adaptation, distribution and reproduction in any medium or format, as long as you give appropriate credit to the original author(s) and the source, provide a link to the Creative Commons licence, and indicate if changes were made. The images or other third party material in this article are included in the article's Creative Commons licence, unless indicated otherwise in a credit line to the material. If material is not included in the article's Creative Commons licence and your intended use is not permitted by statutory regulation or exceeds the permitted use, you will need to obtain permission directly from the copyright holder. To view a copy of this licence, visit http://creativecommons.org/licenses/by/4.0/.

\section{References}

1. Milliron, D.J., Hughes, S.M., Cui, Y., Manna, L., Li, J., Wang, L., Alivisatos, A.P.: Colloidal nanocrystal heterostructures with linear and branched topology. Nature (2004). https://doi. org/10.1038/nature02695

2. Kerker, M.: The optics of colloidal silver: something old and something new. J. Colloid Interface Sci. (1985). https://doi. org/10.1016/0021-9797(85)90304-2

3. Zhang, S., Tang, Y., Vlahovic, B.: A review on preparation and applications of silver-containing nanofibers. Nanoscale Res. Lett. (2016). https://doi.org/10.1186/s11671-016-1286-z

4. Chanel, T.H., Adri, H., Muhammad, D.B., Bambang, Y., Fakhili, G.: Green synthesis of silver nanoparticle and its antibacterial activity. Rasayan J. Chem. (2017). https://doi.org/10.7324/ rjc.2017.1041875

5. Choudhury, R., Majumder, M., Roy, D.N., Basumallick, S., Misra, T.K.: Phytotoxicity of Ag nanoparticles prepared by biogenic and chemical methods. Int. Nano Lett. (2016). https://doi. org/10.1007/s40089-016-0181-z

6. Kharissova, O.V., Dias, H.R., Kharisov, B.I., Pérez, B.O., Pérez, V.M.: The greener synthesis of nanoparticles. Trends Biotechnol. (2013). https://doi.org/10.1016/j.tibtech.2013.01.003 
7. Archna, H.R.: A review on green synthesis of silver nanoparticle, characterization and optimization parameters. Int. J. Res. Eng. Technol. (2016). https://doi.org/10.15623/ijret.2016.0527010

8. Sithara, R., Selvakumar, P., Arun, C., Anandan, S., Sivashanmugam, P.: Economical synthesis of silver nanoparticles using leaf extract of Acalypha hispida and its application in the detection of Mn(II) ions. J. Adv. Res. (2017). https://doi.org/10.1016/j. jare.2017.07.001

9. Mohamed, N.H., Ismail, M.A., Abdel-Mageed, W.M., Shoreit, A.A.: Antimicrobial activity of latex silver nanoparticles using Calotropis procera. Asian Pac. J. Trop. Biomed. (2014). https:// doi.org/10.12980/apjtb.4.201414b216

10. Chauhan, N., Tyagi, A.K., Kumar, P., Malik, A.: Antibacterial potential of Jatropha curcas synthesized silver nanoparticles against food borne pathogens. Front. Microbiol. (2016). https:// doi.org/10.3389/fmicb.2016.01748

11. Alsalhi, M., Devanesan, S., Alfuraydi, A., Vishnubalaji, R., Munusamy, M.A., Murugan, K., et al.: Green synthesis of silver nanoparticles using Pimpinella anisum seeds: Antimicrobial activity and cytotoxicity on human neonatal skin stromal cells and colon cancer cells. Int. J. Nanomed. (2016). https://doi. org/10.2147/ijn.s113193

12. Jayaprakash, N., Vijaya, J.J., Kaviyarasu, K., Kombaiah, K., Kennedy, L.J., Ramalingam, R.J., et al.: Green synthesis of Ag nanoparticles using Tamarind fruit extract for the antibacterial studies. J. Photochem. Photobiol. B Biol. (2017). https://doi. org/10.1016/j.jphotobiol.2017.03.013

13. Rashid, M.I., Mujawar, L.H., Rehan, Z.A., Qari, H., Zeb, J., Almeelbi, T., Ismail, I.M.: One-step synthesis of silver nanoparticles using Phoenix dactylifera leaves extract and their enhanced bactericidal activity. J. Mol. Liq. (2016). https://doi. org/10.1016/j.molliq.2016.09.030

14. Rao, K., Aziz, S., Roome, T., Razzak, A., Sikandar, B., Jamali, K.S., Imran, M., Jabri, T., Shah, M.R.: Gum acacia stabilized silver nanoparticles based nano-cargo for enhanced antiarthritic potentials of hesperidin in adjuvant induced arthritic rats. Artif. Cells Nanomed. Biotechnol. (2018). https://doi. org/10.1080/21691401.2018.1431653

15. Otunola, G., Afolayan, A., Ajayi, E., Odeyemi, S.: Characterization, antibacterial and antioxidant properties of silver nanoparticles synthesized from aqueous extracts of Allium sativum, Zingiber officinale, and Capsicum frutescens. Pharmacogn. Mag. (2017). https://doi.org/10.4103/pm.pm_430_16

16. Nazeruddin, G., Prasad, N., Prasad, S., Shaikh, Y., Waghmare, S., Adhyapak, P.: Coriandrum sativum seed extract assisted in situ green synthesis of silver nanoparticle and its anti-microbial activity. Ind. Crops Prod. (2014). https://doi.org/10.1016/j.inder op.2014.05.040

17. Nasiri, S., Nasiri, S.: Biosynthesis of Silver Nanoparticles Using Carum carvi Extract and its Inhibitory Effect on Growth of Candida albicans. Avicenna J. Med. Biochem. (2016). https://doi. org/10.17795/ajmb-37504

18. Marslin, G., Dias, A.C., Selvakesavan, R.K., Gregory, F., Sarmento, B.: Antimicrobial activity of cream incorporated with silver nanoparticles biosynthesized from Withania somnifera. Int. J. Nanomed. (2015). https://doi.org/10.2147/IJN.S81271

19. Zulfiqar, H., Ayesha, Z., Rasheed, N., Ali, Z., Mehmood, K., Mazher, A., Mahmood, N.: Synthesis of silver nanoparticles using Fagonia cretica and their antimicrobial activities. Nanoscale Adv. (2019). https://doi.org/10.1039/C8NA00343B

20. Dhand, V., Soumya, L., Bharadwaj, S., Chakra, S., Bhatt, D., Sreedhar, B.: Green synthesis of silver nanoparticles using Coffea arabica seed extract and its antibacterial activity. Mater. Sci. Eng. (2016). https://doi.org/10.1016/j.msec.2015.08.018
21. Ahmed, S., Saifullah, Ahmad, M., Swami, B. L., Ikram, S.: Green synthesis of silver nanoparticles using Azadirachta indica aqueous leaf extract. J. Radiat. Res. Appl. Sci. (2016). https://doi. org/10.1016/j.jrras.2015.06.006

22. Prabhu, S., Poulose, E.K.: Silver nanoparticles: mechanism of antimicrobial action, synthesis, medical applications, and toxicity effects. Int. Nano Lett. (2012). https://doi. org/10.1186/2228-5326-2-32

23. Srikar, S.K., Giri, D.D., Pal, D.B., Mishra, P.K., Upadhyay, S.N.: Green synthesis of silver nanoparticles: a review. Green Sustain. Chem. 6, 34-56 (2016). https://doi.org/10.4236/gsc.2016.61004

24. Mata, R., Nakkala, J.R., Sadras, S.R.: Biogenic silver nanoparticles from Abutilon indicum: their antioxidant, antibacterial and cytotoxic effects in vitro. Colloids Surf. B Biointerfaces (2015). https://doi.org/10.1016/j.colsurfb.2015.01.052

25. Sulthana, R.N., Rajanikanth, A.: Green synthesis of silver nanoparticles using seed extract of Foeniculum vulgare and their antibacterial activity. Int. J. Curr. Res. Biosci. Plant Biol. 5(7), 77-83 (2018). https://doi.org/10.20546/ijcrbp.2018.507.010

26. Roy, P., Das, B., Mohanty, A., Mohapatra, S.: Green synthesis of silver nanoparticles using Azadirachta indica leaf extract and its antimicrobial study. Appl. Nanosci. (2017). https://doi. org/10.1007/s13204-017-0621-8

27. Bhuvaneswari, T.S., Thirugnanam, T., Thirumurugan, V.: Phytomediated synthesis of silver nanoparticles using Cassia auriculata L.: evaluation of antibacterial and antifungal activity. Asian J. Pharm. Pharmacol. 5(2), 326-331 (2019). https:// doi.org/10.31024/ajpp.2019.5.2.16

28. Krishnan, V., Bupesh, G., Manikandan, E., Thanigai, A.K., Magesh, S., Kalyanaraman, R., Maaza, M.: Green synthesis of silver nanoparticles using Piper nigrum concoction and its anticancer activity against MCF-7 and Hep-2 cell lines. J. Antimicrob. Agents (2016). https://doi.org/10.4172/2472-1212.10001 23

29. Singh, K., Panghal, M., Kadyan, S., Yadav, J.P.: Evaluation of antimicrobial activity of synthesized silver nanoparticles using Phyllanthus amarus and Tinospora cordifolia medicinal plants. J. Nanomed. Nanotechnol. (2014). https://doi.org/10.4172/21577439.1000250

30. Prasad, T., Elumalai, E.: Biofabrication of Ag nanoparticles using Moringa oleifera leaf extract and their antimicrobial activity. Asian Pac. J. Trop. Biomed. (2011). https://doi.org/10.1016/ s2221-1691(11)60096-8

31. Providence, B.A., Chinyere, A.A., Ayi, A.A., Charles, O.O., Elijah, T.A., Ayomide, H.L.: Green synthesis of silver monometallic and copper-silver bimetallic nanoparticles using Kigelia africana fruit extract and evaluation of their antimicrobial activities. Int. J. Phys. Sci. 13(3), 24-32 (2018). https://doi.org/10.5897/ijps2 017.4689

32. Kumar, C., Yugandhar, P., Savithramma, N.: Biological synthesis of silver nanoparticles from Adansonia digitata L. fruit pulp extract, characterization, and its antimicrobial properties. J. Intercult. Ethnopharmacol. (2016). https://doi.org/10.5455/jice.20160 124113632

33. Kumar, C.M.K., Yugandhar, P., Savithramma, N.: Adansonia digitata leaf extract mediated synthesis of silver nanoparticles; characterization and antimicrobial studies. J. Appl. Pharm. Sci. (2015). https://doi.org/10.7324/JAPS.2015.50813

34. Ali, K., Ahmed, B., Dwivedi, S., Saquib, Q., Al-Khedhairy, A.A., Musarrat, J.: Microwave accelerated green synthesis of stable silver nanoparticles with Eucalyptus globulus leaf extract and their antibacterial and antibiofilm activity on clinical isolates. PLoS One (2015). https://doi.org/10.1371/journal.pone.0131178

35. Muthu, K., Priya, S.: Green synthesis, characterization and catalytic activity of silver nanoparticles using Cassia auriculata 
flower extract separated fraction. Spectrochim. Acta Part A Mol. Biomol. Spectrosc. (2017). https://doi.org/10.1016/j. saa.2017.02.024

36. Khalil, M.M., Ismail, E.H., El-Baghdady, K.Z., Mohamed, D.: Green synthesis of silver nanoparticles using olive leaf extract and its antibacterial activity. Arab. J. Chem. (2014). https://doi. org/10.1016/j.arabjc.2013.04.007

37. Sahni, G., Panwar, A., Kaur, B.: Controlled green synthesis of silver nanoparticles by Allium cepa and Musa acuminata with strong antimicrobial activity. Int. Nano Lett. (2015). https://doi. org/10.1007/s40089-015-0142-y

38. Sathyavathi, R., Krishna, M.B., Rao, S.V., Saritha, R., Rao, D.N.: Biosynthesis of silver nanoparticles using Coriandrum sativum leaf extract and their application in nonlinear optics. Adv. Sci. Lett. (2010). https://doi.org/10.1166/as1.2010.1099

39. Venugopal, N., Mitra, A.: Influence of temperature dependent morphology on localized surface plasmon resonance in ultrathin silver island films. Appl. Surf. Sci. (2013). https://doi. org/10.1016/j.apsusc.2013.08.062

40. Usmani, A., Mishra, A., Jafri, A., Arshad, M., Siddiqui, M.A.: Green synthesis of silver nanocomposites of Nigella sativa seeds extract for hepatocellular carcinoma. Curr. Nanomater. (2019). https://doi.org/10.2174/2468187309666190906130115

41. Kumar, B., Smita, K., Cumbal, L., Debut, A.: Green synthesis of silver nanoparticles using Andean blackberry fruit extract. Saudi J. Biol. Sci. 24(1), 45-50 (2017). https://doi. org/10.1016/j.sjbs.2015.09.006

42. Sundeep, D., Kumar, T.V., Rao, P.S., Ravikumar, R.V.S.S.N., Krishna, A.G.: Green synthesis and characterization of Ag nanoparticles from Mangifera indica leaves for dental restoration and antibacterial applications. Progr. Biomater. (2017). https://doi.org/10.1007/s40204-017-0067-9

43. Eustis, S., El-Sayed, M.A.: Why gold nanoparticles are more precious than pretty gold: noble metal surface plasmon resonance and its enhancement of the radiative and nonradiative properties of nanocrystals of different shapes. ChemInform (2006). https://doi.org/10.1002/chin.200625211

44. Ajitha, B., Reddy, Y.A., Reddy, P.S.: Green synthesis and characterization of silver nanoparticles using Lantana camara leaf extract. Mater. Sci. Eng. (2015). https://doi.org/10.1016/j. msec.2015.01.035

45. Usha, C., Gladys, A.R.: Biogenic synthesis of silver nanoparticles by Acacia nilotica and their antibacterial activity. Int. J. Sci. Res. 3(6), 27-29 (2012). https://doi.org/10.15373/22778 179/june2014/11

46. Uzunuigbe, E., Mlowe, S., Revaprasadu, N., Kappo, A.P.: Synthesis, characterization and antibacterial activity of silver nanoparticles using Acacia senegal leaf extract. Paper Present. Past Present Res. Syst. Green Chem. (2016). https://doi. org/10.4172/2161-0401.C1.013

47. Tan, G.: Green synthesis of silver nanoparticles using Allium cepa and Allium sativum extract: a comparative characterization study. J. Biotechnol. (2017). https://doi.org/10.1016/j.jbiot ec.2017.06.669

48. Ahlawat, J., Sehrawat, A.R.: Nano Dimensional (1-20 nm) Silver nanoparticles: stem extract of Capparis decidua (FORSK) EDGEW mediated synthesis and its characterization-a lab to land approach. Int. J. Curr. Microbiol. Appl. Sci. (2017). https ://doi.org/10.20546/ijcmas.2017.610.226

49. Gondwal, M., Pant, G.J.: Synthesis and catalytic and biological activities of silver and copper nanoparticles using Cassia occidentalis. Int. J. Biomater. (2018). https://doi. org/10.1155/2018/6735426

50. Ponarulselvam, S., Panneerselvam, C., Murugan, K., Aarthi, N., Kalimuthu, K., Thangamani, S.: Synthesis of silver nanoparticles using leaves of Catharanthus roseus Linn. G. Don and their antiplasmodial activities. Asian Pac. J. Trop. Biomed. (2012). https://doi.org/10.1016/s2221-1691(12)60100-2

51. Logeswari, P., Silambarasan, S., Abraham, J.: Synthesis of silver nanoparticles using plants extract and analysis of their antimicrobial property. J. Saudi Chem. Soc. (2015). https://doi. org/10.1016/j.jscs.2012.04.007

52. Syafiuddin, A., Salmiati, Hadibarata, T., Kueh, A.B., Salim, M.R.: Novel weed-extracted silver nanoparticles and their antibacterial appraisal against a rare bacterium from river and sewage treatment plan. Nanomaterials (2017). https://doi. org/10.3390/nano8010009

53. Gomathi, M., Rajkumar, P., Prakasam, A., Ravichandran, K.: Green synthesis of silver nanoparticles using Datura stramonium leaf extract and assessment of their antibacterial activity. Resour. Effic. Technol. (2017). https://doi.org/10.1016/j.reffi t.2016.12.005

54. Thovhogi, N., Diallo, A., Gurib-Fakim, A., Maaza, M.: Nanoparticles green synthesis by Hibiscus Sabdariffa flower extract: main physical properties. J. Alloys Compd. (2015). https://doi. org/10.1016/j.jallcom.2015.06.076

55. Patil, S.P., Kumbhar, S.T.: Antioxidant, antibacterial and cytotoxic potential of silver nanoparticles synthesized using terpenes rich extract of Lantana camara L. leaves. Biochem. Biophys. Rep. (2017). https://doi.org/10.1016/j.bbrep.2017.03.002

56. Gupta, A., Ingle, A., Gade, A.K., Gaikwad, S., Rai, M., Bonde, S.R.: Lawsonia inermis-mediated synthesis of silver nanoparticles: activity against human pathogenic fungi and bacteria with special reference to formulation of an antimicrobial nanogel. IET Nanobiotechnol. (2014). https://doi.org/10.1049/ iet-nbt.2013.0015

57. Bharathi, V., Jannathul, F., Noorzaid, M., Resni, M.: Green synthesis of Mangifera indica silver nanoparticles and its analysis using Fourier transform infrared and scanning electron microscopy. Natl. J. Physiol. Pharm. Pharmacol. (2017). https://doi. org/10.5455/njppp.2017.7.0725428082017

58. Khatoon, A., Khan, F., Ahmad, N., Shaikh, S., Rizvi, S.M., Shakil, S., et al.: Silver nanoparticles from leaf extract of Mentha piperita: Eco-friendly synthesis and effect on acetylcholinesterase activity. Life Sci. (2018). https://doi.org/10.1016/j. lfs.2018.08.046

59. Moodley, J.S., Krishna, S.B., Pillay, K., Sershen, Govender, P.: Green synthesis of silver nanoparticles from Moringa oleifera leaf extracts and its antimicrobial potential. Adv. Nat. Sci. Nanosci. Nanotechnol. (2018). https://doi.org/10.1088/20436254/aaabb2

60. Ajitha, B., Reddy, Y.A., Jeon, H., Ahn, C.W.: Synthesis of silver nanoparticles in an eco-friendly way using Phyllanthus amarus leaf extract: antimicrobial and catalytic activity. Adv. Powder Technol. (2018). https://doi.org/10.1016/j.apt.2017.10.015

61. Ojha, S., Sett, A., Bora, U.: Green synthesis of silver nanoparticles by Ricinus communis var. carmencita leaf extract and its antibacterial study. Adv. Nat. Sci. Nanosci. Nanotechnol. 8(3), 035009 (2017). https://doi.org/10.1088/2043-6254/aa724b

62. Gopinath, V., Mubarakali, D., Priyadarshini, S., Priyadharsshini, N.M., Thajuddin, N., Velusamy, P.: Biosynthesis of silver nanoparticles from Tribulus terrestris and its antimicrobial activity: a novel biological approach. Colloids Surf. B Biointerfaces (2012). https://doi.org/10.1016/j.colsurfb.2012.03.023

63. Rajesh, P., Swati, W., Sandesh, M., Sangita, J., Kulkarni, S.: Green synthesis of silver nanoparticles by Withania somnifera and evaluation of its antimicrobial potential. J. Empir. Biol. 1(2), 38-48 (2013). https://doi.org/10.13074/jent.2013.02.121028

64. Ynalvez, R., Compean, K.: Antimicrobial activity of plant secondary metabolites: a review. Res. J. Med. Plant (2014). https:// doi.org/10.3923/rjmp.2014.204.213 
65. Jain, S., Mehata, M.S.: Medicinal plant leaf extract and pure flavonoid mediated green synthesis of silver nanoparticles and their enhanced antibacterial property. Sci. Rep. (2017). https:// doi.org/10.1038/s41598-017-15724-8

66. Ahmad, S., Munir, S., Zeb, N., Ullah, A., Khan, B., Ali, J., et al.: Green nanotechnology: a review on green synthesis of silver nanoparticles - an ecofriendly approach. Int. J. Nanomed. 14, 5087-5107 (2019). https://doi.org/10.2147/ijn.s200254

67. Siddiqi, K.S., Husen, A.: Fabrication of metal nanoparticles from fungi and metal salts: scope and application. Nanoscale Res. Lett. (2016). https://doi.org/10.1186/s11671-016-1311-2

68. Adeeyo, A.O., Odiyo, J.O.: Biogenic synthesis of silver nanoparticle from mushroom exopolysaccharides and its potentials in water purification. Open Chem. J. 5(1), 64-75 (2018). https:// doi.org/10.2174/1874842201805010064

69. Ghorbani, H.R.: Biosynthesis of silver nanoparticles by Escherichia coli. Asian J. Chem. (2013). https://doi.org/10.14233/ajche m.2013.12805

70. Ilavarasan, R., Vadivelu, L.: Phytochemical and quality assessment of Acacia nilotica Linn and Acacia leucophloea willd flowers. Pharmacogn. J. (2017). https://doi.org/10.5530/pj.2017.6.113

71. Okoro, S.O., Kawo, A.H., Arzai, A.H.: Phytochemical screening, antibacterial and toxicological activities of Acacia senegal extracts. Bayero J. Pure Appl. Sci. 5(1), 163-170 (2012). https ://doi.org/10.4314/bajopas.v5i1.29

72. Eltayeb, I.M., Elhassan, I.A., Elrasoul, J.H., Eldind, E.S.: A comparative study of chemical composition of Acacia Seyal stem, stem wood and stem bark dry distillates used by sudanese women as cosmetic and medicine. Int. J. Pharm. Pharm. Sci. (2017). https://doi.org/10.22159/ijpps.2017v9i11.21802

73. Arora, E., Sharma, V., Khurana, A., Manchanda, A., Sahani, D., Abraham, S., Jomy, S.: Phytochemical analysis and evaluation of antioxidant potential of ethanol extract of Allium cepa and ultrahigh homoeopathic dilutions available in the market: a comparative study. Indian J. Res. Homoeopath. 11(2), 88 (2017). https:// doi.org/10.4103/ijrh.ijrh_13_17

74. Yasmin, H., Anbumalarmathi, J., Sharmili, S.A.: Phytochemical analysis and antimicrobial activity of garlic (Allium sativum L.) and onion (Allium cepa L.). Res. Crops 19(2), 245 (2018). https ://doi.org/10.5958/2348-7542.2018.00035.9

75. Offor, C.: Comparative chemical analyses of Vernonia amygdalina and Azadirachta indica leaves. IOSR J. Pharm. Biol. Sci. 9(5), 73-77 (2014). https://doi.org/10.9790/3008-09527377

76. Oke, D.G.: Proximate and phytochemical analysis of Cajanus cajan (Pigeon Pea) leaves. Chem. Sci. Trans. (2014). https://doi. org $/ 10.7598 /$ cst 2014.785

77. Morsy, N., Sherif, E.A., Abdel-Rassol, T.M.: Phytochemical analysis of Calotropis procera with antimicrobial activity investigation. Main Group Chem. (2016). https://doi.org/10.3233/ mgc-160206

78. Zia-Ul-Haq, M., Ćavar, S., Qayum, M., Imran, I., Feo, V.D.: Compositional studies: antioxidant and antidiabetic activities of Capparis decidua (Forsk.) Edgew. Int. J. Mol. Sci. (2011). https ://doi.org/10.3390/ijms12128846

79. Nascimento, P., Nascimento, T., Ramos, N., Silva, G., Gomes, J., Falcão, R., Silva, T.: Quantification, antioxidant and antimicrobial activity of phenolics isolated from different extracts of Capsicum frutescens (Pimenta Malagueta). Molecules (2014). https://doi.org/10.3390/molecules19045434

80. Barkat, M.Q., Mahmood, H.K.: Phytochemical And Antioxidant Screening Of Zingiber officinale, Piper nigrum, Rutag raveolanes and Carum carvi And Their Effect On Gastrointestinal Tract Activity. Matrix Science Medica (2018). https://doi. org/10.26480/msm.01.2018.09.13
81. Asgarpanah, J.: Phytochemistry, pharmacology and medicinal properties of Coriandrum sativum L. Afr. J. Pharm. Pharmacol. (2012). https://doi.org/10.5897/ajpp12.901

82. Murugan, M., Murugan, T., Wins, J.: Antimicrobial activity and phytochemical constituents of leaf extracts of Cassia auriculata. Indian J. Pharm. Sci. 75(1), 122 (2013). https://doi. org/10.4103/0250-474x.113546

83. Srividya, S., Sridevi, G., Manimegalai, A.G.: Phytochemical screening and in vitro antioxidant activity of ethanolic extract of Cassia occidentalis. Int. J. Pharm. Clin. Res. (2017). https://doi. org/10.25258/ijpcr.v9i3.8327

84. Rajalakshmi, B.G., Komathi, S.K.S., Poongodi, N., Sasikala, T., Banuraviganesh, B.: Antimicrobial activity and phytochemical screening of Catharanthus roseus. Int. J. Sci. Res. 2(10), 1-2 (2012). https://doi.org/10.15373/22778179/oct2013/156

85. Favela-Hernández, J., González-Santiago, O., Ramírez-Cabrera, M., Esquivel-Ferriño, P., Camacho-Corona, M.: Chemistry and pharmacology of Citrus sinensis. Molecules 21(2), 247 (2016). https://doi.org/10.3390/molecules21020247

86. Block, S.: Diterpenes from the leaves of Croton zambesicus. Phytochemistry 65(8), 1165-1171 (2004). https://doi.org/10.1016/j. phytochem.2004.02.023

87. Lawal, O., Oyedeji, A.: Chemical composition of the essential oils of Cyperus rotundus L. from South Africa. Molecules (2009). https://doi.org/10.3390/molecules14082909

88. Soni, P., Siddiqui, A.A., Dwivedi, J., Soni, V.: Pharmacological properties of Datura stramonium L. as a potential medicinal tree: an overview. Asian Pac. J. Trop. Biomed. 2(12), 1002-1008 (2012). https://doi.org/10.1016/s2221-1691(13)60014-3

89. Saeed, M.A., Sabir, A.W.: Effects of Fagonia cretica L. constituents on various haematological parameters in rabbits. J. Ethnopharmacol. 85(2-3), 195-200 (2003). https://doi.org/10.1016/ s0378-8741(02)00365-3

90. Badgujar, S.B., Patel, V.V., Bandivdekar, A.H.: Foeniculum vulgare Mill: a review of its botany, phytochemistry, pharmacology, contemporary application, and toxicology. Biomed. Res. Int. (2014). https://doi.org/10.1155/2014/842674

91. Tomar, N.S., Sharma, M., Agarwal, R.M.: Phytochemical analysis of Jatropha curcas L. during different seasons and developmental stages and seedling growth of wheat (Triticum aestivum L.) as affected by extracts/leachates of Jatropha curcas L. Physiol. Mol. Biol. Plants 21(1), 83-92 (2014). https://doi. org/10.1007/s12298-014-0272-0

92. Bello, I., Shehu, M.W., Musa, M., Asmawi, M.Z., Mahmud, R.: Kigelia africana (Lam.) Benth. (Sausage tree): phytochemistry and pharmacological review of a quintessential African traditional medicinal plant. J. Ethnopharmacol. 189, 253-276 (2016). https://doi.org/10.1016/j.jep.2016.05.049

93. Verma, R.K., Verma, S.K.: Phytochemical and termiticidal study of Lantana camara var. aculeata leaves. Fitoterapia (2006). https ://doi.org/10.1016/j.fitote.2006.05.014

94. Li, Q., Gao, W.Q., Zhao, Y.Q.: Advances in studies on chemical constituents and biological activities of Lawsonia inermis. China J. Chin. Mater. Med. (2013). https://doi.org/10.4268/ cjcmm20130604

95. Adesegun, S., Ayoola, G., Coker, H., Adepoju-Bello, A., Obaweya, K., Ezennia, E., Atangbayila, T.: Phytochemical screening and antioxidant activities of some selected medicinal plants used for malaria therapy in Southwestern Nigeria. Trop. J. Pharm. Res. (2008). https://doi.org/10.4314/tjpr.v7i3.14686

96. Sujana, P., Sridhar, T.M., Josthna, P., Naidu, C.V.: Antibacterial activity and phytochemical analysis of Mentha piperita L. (Peppermint)—an important multipurpose medicinal plant. Am. J. Plant Sci. (2013). https://doi.org/10.4236/ajps.2013.41012

97. Leone, A., Spada, A., Battezzati, A., Schiraldi, A., Aristil, J., Bertoli, S.: Cultivation, genetic, ethnopharmacology, phytochemistry 
and pharmacology of Moringa oleifera leaves: an overview. Int. J. Mol. Sci. (2015). https://doi.org/10.3390/ijms160612791

98. Javed, S.: Nutritional, phytochemical potential and pharmacological evaluation of Nigella Sativa (Kalonji) and Trachyspermum Ammi (Ajwain). J. Med. Plants Res. (2012). https://doi. org/10.5897/JMPR11.1341

99. Hashmi, M.A., Khan, A., Hanif, M., Farooq, U., Perveen, S.: Traditional uses, phytochemistry, and pharmacology of Olea europaea (Olive). Evid. Based Complement. Altern. Med. (2015). https://doi.org/10.1155/2015/541591

100. Bennaceur, M., Bengag, A., Marouf, A., Bouguedoura, N.: Phytochemical Profile And Antioxidant Activity of Phoenix dactylifera L., Phoenix canariensis L. and Chamaerops humilis L. Acta Hortic. (2010). https://doi.org/10.17660/actahortic.2010.882

101. Oluwafemi, F., Debiri, F.: Antimicrobial effect of Phyllanthus amarus and Parquetina nigrescens on Salmonella typhi. Afr. J. Biomed. Res. (2010). https://doi.org/10.4314/ajbr.v11i2.50712

102. Suurbaar, J., Mosobil, R., Donkor, A.-M.: Antibacterial and antifungal activities and phytochemical profile of leaf extract from different extractants of Ricinus communis against selected pathogens. BMC Res. Notes (2017). https://doi.org/10.1186/ s13104-017-3001-2

103. Abukakar, M., Ukwuani, A., Shehu, R.: phytochemical screening and antibacterial activity of Tamarindus indica pulp extract. Asian J. Biochem. (2008). https://doi.org/10.3923/ ajb.2008.134.138

104. Sinha, A.: Phyto-chemical studies of methanol extracts of Tinospora cordifolia stem by Gc-Ms. World J. Pharm. Res. (2017). https://doi.org/10.20959/wjpr20174-8205

105. Shalini, R., Jolly, K.E., Deepa, M.S.: Physical and phytochemical screening of market samples of ashwagandha [Withania somnifera (Linn) Dunal] in kerala. Int. J. Adv. Res. 5(8), 2018-2024 (2017). https://doi.org/10.21474/ijar01/5268

Publisher's Note Springer Nature remains neutral with regard to jurisdictional claims in published maps and institutional affiliations. 